\title{
SYMMETRISABLE OPERATORS
}

\author{
PART III \\ HILBERT SPAGE OPERATORS SYMMETRISABLE \\ BY BOUNDED OPERATORS
}

J. P. O. SILBERSTEIN

(received 8 February 1961, revised 30 October 1962)

\section{Introduction}

The fact that the most general symmetrisable operators in Hilbert Space do not possess a number of the desirable properties of such operators in unitary spaces makes it necessary to look for a more restricted class of operators. There are two reasons for our particular choice. In the first place many of the conditions introduced in the course of Part II concerned relationships between the domain of the symmetrising operator $H$ and the domain and range of the symmetrisable operator $A$. These conditions are now all automatically satisfied. The other reason is that the construction used in section 4 to relate symmetrisable operators to certain symmetric operators clearly required that either $H$ or $H^{-1}$ was bounded. The case of $H^{-1}$ bounded has already been dealt with in section 9 and shown to be fairly simple. The case in which $H$ is bounded is clearly of considerable complexity, since we have already seen (example in proof of Theorem 10.6.) that the continuous spectrum may be complex in this case. We follow the usual convention and define $|H|$ the bound of $H$ by

$$
|H|=\sup _{\substack{\|x\|=1 \\ x \in \mathfrak{G}}}\|H x\|
$$

\section{Remarks on the bound of $H$, on $\Re_{H}$ and some properties of $A$ already established}

We can assume that the bound of $H$ is equal to 1 . This is because if $H$ is a symmetrising operator, so is $\alpha H$ where $\alpha$ is any real positive number, in particular $\alpha=1 /(b d$ of $H)$.

For simplicity we shall assume $\mathfrak{N}_{H}=[0]$, i.e. $H$ is strictly positive. We are already aware of the complication which arises when $\mathfrak{R}_{H}$ has positive dimension. On the other hand we can proceed from the general case to ours 
as can be seen from the following argument. The null-spaces are related by $\mathfrak{N}_{H} \subset \mathfrak{R}_{A} \subset \mathfrak{R}_{H A} ; \mathfrak{N}_{H}$ is closed and $\mathfrak{N}_{H}^{\perp}$ is a closed subspace say $\mathfrak{M}$. Let $P_{\mathfrak{R}}$ be the projector on $\mathfrak{M}$. Then since $\mathfrak{N}_{H}$ reduces both $H$ and $H A$, $\mathfrak{R}$ does likewise. It follows that $H A=P_{\mathrm{MR}} H A P_{\mathfrak{M}}=P_{\mathfrak{M}} H P_{\mathfrak{M}} P_{\mathfrak{M}} A P_{\mathfrak{M R}}$. Let $H_{\mathfrak{M R}}$ and $A_{\mathfrak{M}}$ be the restrictions of $P_{\mathfrak{M}} H P_{\mathfrak{M}}$ and $P_{\mathfrak{M}} A P_{\mathfrak{M}}$ to subspace $\mathbb{M}$. Then on $\mathfrak{M} H_{\mathfrak{R}}$ is strictly positive and symmetrises $A_{\mathfrak{R}} \cdot \mathfrak{M}$ is a Hilbert Space or a Unitary Space so that the theory of operators symmetrisable by positive operators is equivalent to the theory of $P_{\mathfrak{M}} A P_{\mathfrak{M}}$ (or its restriction on $\mathfrak{M}$ ) where $A$ is any symmetrisable operator.

In the remainder of this paper it will be assumed (unless the contrary is stated) that $H$ is a strictly positive definite self-adjoint bounded linear operator in $\mathfrak{g}$ with upper bound 1 at most. Unless otherwise stated $H A$ is assumed self-adjoint, i.e. the operators $A$ are assumed symmetrisable in the strict sense.

The results obtained in Part II showed that any symmetrisable $A$ under consideration will be closed and $A=A^{+*}=A^{* *}$. Further the point spectrum of $A$ and of $A^{*}$ (if it exists) is real. (This is no longer true for $A^{*}$ if $H A$ is merely symmetric). The continuous spectrum may be complex and we shall investigate conditions under which it is real.

Remark 11.1. The continuous spectrum of a closed linear operator $A$ is usually defined as consisting of those points $\lambda$ for which $A_{\lambda}^{-1} \equiv(A-\lambda I)^{-1}$ is defined as an unbounded closed linear operator on a dense domain. It follows from this that for every $y$ in $\mathfrak{W}$, with $\|y\|=1$ say, there exists for any $\varepsilon_{n}>0$ a $y_{n}$, with $\left\|y_{n}\right\|=1$ and $\left\|y-y_{n}\right\| \leqq \varepsilon_{n}$ such that $y_{n}$ is in the domain of $A_{\lambda}^{-1}$ with $\left\|A_{\lambda}^{-1} y_{n}\right\| \geqq M_{n}\left\|y_{n}\right\|$ where $M_{n}$ is arbitrarily large. Thus if $y \notin \Re_{A_{\lambda}}$ there exists a sequence $\left(y_{n}\right)$ tending to $y$ such that $x_{n}=A_{\lambda}^{-1} y_{n}$ with $\left\|x_{n}\right\| \rightarrow \infty$, in fact every sequence tending to $y$ contains such a subsequence (Cf. [1] theorem 3.17). Putting $z_{n}=x_{n} /\left\|x_{n}\right\|$ we have a sequence $\left(z_{n}\right)$ such that

$$
\begin{aligned}
A_{\lambda} z_{n} & =\frac{1}{\left\|x_{n}\right\|} y_{n} \\
& =\varepsilon_{n} y_{n}
\end{aligned}
$$

where $\left\|z_{n}\right\|=\left\|y_{n}\right\|=1$ and $\varepsilon_{n} \rightarrow 0$ as $n \rightarrow \infty\left(\left(z_{n}\right)\right.$ does not tend to a limit because $\lambda$ would otherwise be an eigenvalue since $A_{\lambda}$ is closed). The existence of sequences such as $\left(z_{n}\right)$ is therefore a necessary condition for $\lambda$ to belong to the continuous spectrum.

\section{A special class of operators}

In order to show that it is plausible to enquire further into the continuous spectrum we discuss operators of the type $B H$ where $B, H$ are 
self-adjoint and bounded and $H$ is positive definite. Such operators are clearly symmetrisable by $\alpha H$ where $\alpha$ is any positive real number. We can prove

THEOREM 12.1. If $H$ is positive definite and $H, B$ are bounded seltadjoint operators then the continuous spectrum of $A=B H$ cannot include a point not on the real axis.

Proor. Since $B H=(|H| B)\left(|H|^{-1} H\right)$ where $|H|$ is the bound of $H$, we can take the bound of $H$ to be 1 without loss of generality. Next we suppose $\lambda$ to be in the continuous spectrum; then by Remark 11.1 there exists a sequence $\left(x_{n}\right)$ such that

$$
A x_{n}-\lambda x_{n}=\varepsilon_{n} y_{n}
$$

where $\left\|x_{n}\right\|=\left\|y_{n}\right\|=1 ; \varepsilon_{n} \rightarrow 0$ as $n \rightarrow \infty$. ( $\varepsilon_{n}$ can be taken real, positive).

Let $H x_{n}=\alpha x_{n}+\beta z_{n}$ where $\alpha(>0), \beta$ are real and $z_{n}$ is an element of unit length orthogonal to $x_{n}$. (We can clearly choose $z_{n}$ so that $\beta$ is real; $\alpha$ is real by the self-adjointness of $H)$. Let $\left(x_{n}, z_{n}, u_{n}\right)$ be a complete orthonormal set in the subspace spanned by $\left(x_{n}, z_{n}, y_{n}\right)$. Further, for some real $b_{11}, b_{22}$ and complex $b_{i j}(i \neq j)$ depending on $n$ but such that $\left|b_{i j}\right| \leqq|B|$,

$$
\begin{aligned}
& B x_{n}=b_{11} x_{n}+b_{12} z_{n}+\delta_{13} u_{n}+b_{14} v_{n} \\
& B z_{n}=b_{12} x_{n}+b_{22} z_{n}+b_{23} u_{n}+\delta_{24} v_{n}+\delta_{25} w_{n}
\end{aligned}
$$

where $v_{n}, w_{n}$ complete the orthonormal set in $\left\{x_{n}, z_{n}, B x_{n}, B z_{n}, y_{n}\right\}$. Also

$$
y_{n}=\gamma_{1} x_{n}+\gamma_{2} z_{n}+\gamma_{3} u_{n}
$$

for some numbers $\gamma_{i}$ such that $\left|\gamma_{i}\right| \leqq 1(i=1,2,3)$. Then

Hence

$$
\begin{aligned}
B H x_{n}= & \left(\alpha b_{11}+\beta b_{12}\right) x_{n}+\left(\alpha b_{12}+\beta b_{22}\right) z_{n} \\
& +\left(\alpha b_{13}+\beta b_{23}\right) u_{n}+\left(\alpha b_{14}+\beta b_{24}\right) v_{n} \\
& +\beta b_{25} w_{n} \\
= & \lambda x_{n}+\varepsilon_{n} \gamma_{1} x_{n}+\varepsilon_{n} \gamma_{2} z_{n}+\varepsilon_{n} \gamma_{3} u_{n} .
\end{aligned}
$$

$$
\begin{aligned}
& \alpha b_{11}+\beta b_{12}=\lambda+\varepsilon_{n} \gamma_{1}=\lambda^{\prime} \text { say, } \\
& \alpha b_{12}+\beta b_{22}=\varepsilon_{n} \gamma_{2}, \\
& \alpha b_{13}+\beta b_{23}=\varepsilon_{n} \gamma_{3}, b_{14}=-\beta / \alpha b_{24}, b_{25}=0 .
\end{aligned}
$$

Now we suppose the imaginary part of $\lambda, \mathscr{I}(\lambda) \neq 0$. Then if $n$ is large enough $\left|\mathscr{F}\left(\lambda^{\prime}\right)\right| \geqq|| \mathscr{F}(\lambda)\left|-\varepsilon_{n}\right|>0$ and by (i) $\beta \neq 0$ so that

$$
b_{12}=\left(\lambda^{\prime}-\alpha \beta_{11}\right) / \beta \text {. }
$$


Further by (ii)

so that

$$
\varepsilon_{n} \gamma_{2}=\beta b_{22}+(\alpha / \beta) \lambda^{\prime}-\left(\alpha^{2} / \beta\right) b_{11}
$$

$$
\mathscr{I}\left(\varepsilon_{n} \gamma_{2}\right)=(\alpha / \beta) \mathscr{F}\left(\lambda^{\prime}\right) \text {. }
$$

For $n$ sufficiently large, $\varepsilon_{n}$ is arbitrarily small so that $\alpha / \beta$ is arbitrarily small. Since $H$ is bounded this implies $\alpha$ is arbitrarily small. Let $\gamma=\left(H z_{n}, z_{n}\right)$ then since $|H| \leqq 1, \gamma \leqq 1$. Since $H$ is positive $\beta^{2} \leqq \alpha \gamma<\alpha$. Therefore $\beta$ is arbitrarily small. It follows that $\left(b_{12}\right)=\lambda^{\prime} \mid \beta$ is arbitrarily large, which is impossible since $B$ is bounded. Hence if $\mathscr{S}(\lambda) \neq 0$ it is impossible for $\lambda$ to belong to the continuous spectrum.

The complete statement of the spectral properties of these special operators is

THEOREM 12.2. For operators of type $A=B H$ where $B, H$ are bounded and self-adjoint and $H$ is positive, the point spectrum and continwous spectrum are real, the residual spectrum is empty.

The first two statements were proved in theorems 10.1 and 12.1 To prove the final statement we observe that $A^{*}=H B$ which is symmetrisable by the positive definite operator $H^{-1}$. Hence by theorem 10.9 the result follows.

\section{Two general lemmas and another proof of theorem 12.1}

LEMMA 13.1. If $\left(x_{n}\right)$ is a sequence such that $\left\|x_{n}\right\|=1$ and for some non real $\lambda\left\|A_{\lambda} x_{n}\right\| \rightarrow 0$, then if $H$ symmetrices $A,\left\|H \downarrow x_{n}\right\| \rightarrow 0$.

By assumption there exists a sequence of numbers $\varepsilon_{n}$ tending to 0 and a sequence of elements $y_{n}$ with $\left\|y_{n}\right\|=1$ such that

Therefore

$$
A x_{n}-\lambda x_{n}=\varepsilon_{n} y_{n} \text {. }
$$

$$
\left(H A x_{n}, x_{n}\right)=\lambda\left(H x_{n}, x_{n}\right)+\varepsilon_{n}\left(H y_{n}, x_{n}\right) .
$$

The left hand side is real so that, taking imaginary parts

and therefore

$$
\left\|H^{\mathbf{t}} x_{n}\right\|^{2}=-\frac{\mathscr{S}\left[\varepsilon_{n}\left(H y_{n}, x_{n}\right)\right]}{\mathscr{\sigma}(\lambda)}
$$

$$
\left\|H x_{n}\right\| \leqq \frac{\left\|\varepsilon_{n}\right\|}{|\mathcal{F}(\lambda)|}\left\|H \mathfrak{t} y_{n}\right\| .
$$

Since $H$ is bounded the right hand side tends to 0 with $n$ which establishes the lemma.

An immediate consequence of this is 
Lemma 13.2. The sequence $\left(x_{n}\right)$ of Lemma 13.1. tends weakly to 0 .

Proof. For any $x$ such that $\left\|H^{i} x\right\|=1$

so that

$$
\left(x_{n}, H x\right)=\left(H x_{n}, x\right) \leqq\left\|H^{\frac{1}{2}} x_{n}\right\|
$$

$$
\lim _{n \rightarrow \infty}\left(x_{n}, H x\right)=0 .
$$

Since $\left\|x_{n}\right\|=1$ for all $n$ and the set of all $H x$ is dense in $\mathfrak{S}$ it follows that $x_{n} \rightarrow 0$.

Finally we use the first of these lemmas to give an alternative proof of theorem 12.1. With the notation introduced there let us suppose again that $\mathscr{I}(\lambda) \neq 0$ and let $|B|$ be the bound of $B$. Then $\left\|B H x_{n}\right\| \leqq|B|$ $\left\|H x_{n}\right\| \leqq|B|\left\|H \ddagger x_{n}\right\|$. By equation (12.1)

so that

$$
B H x_{n}=\lambda x_{n}+\varepsilon_{n} y_{n}
$$

$$
\begin{aligned}
\left\|B H x_{n}\right\| & =\left\|\lambda x_{n}+\varepsilon_{n} y_{n}\right\| \\
& \geqq|| \lambda|-| \varepsilon_{n}|| .
\end{aligned}
$$

But by lemma 13.1, if $\mathscr{I}(\lambda) \neq 0,\left\|H^{\frac{1}{2}} x_{n}\right\| \rightarrow 0$ so that by the above $\| B H x_{n}|| \rightarrow 0$, which would require $|\lambda|=0$ contrary to hypothesis.

\section{The relationship between symmetrisable operators and certain self-adjoint operators}

We now proceed to generalise the representation given in section 4 for symmetrisable operators in $\mathfrak{u}_{n}$. We showed there that a symmetrisable operator was related by a process of projection to a Hermitian symmetric or self-adjoint operator. There were two representations, one applying to an operator whose symmetrising operator had lower bound 1 , and the other $-A^{*}$ in that case - with upper bound equal to 1 . Since we are dealing with operators symmetrisable by a bounded operator we shall evidently wish to generalise the procedure adopted for $A^{*}$ in theorem 4.1. We use the following notation:

$\mathfrak{W}$ is the Hilbert Space in which $H$ and $A$ are defined.

$\mathfrak{W}_{2}=\mathfrak{W}+\mathfrak{W}^{\prime}$ is a Hilbert Space containing $\mathfrak{W}, \mathfrak{S}^{\prime}$ as subspaces of "equal dimension" and as orthogonal complements.

$\mathfrak{S}^{\prime}$ orthogonal complement of $\mathfrak{W}$ in $\mathfrak{W}_{2}$. (Is a Hilbert Space isomorphic with (马).

$S$ Symmetric unitary operator (Symmetry for short) in $\mathfrak{W}_{2}$ which transforms $\mathfrak{S}$ into $\mathfrak{S}^{\prime}$ and $\mathfrak{S}^{\prime}$ into $\mathfrak{b}$. 
$H$ Self-adjoint positive definite operator in $\mathfrak{S}$ with bound less than 1 . (The requirement of strict inequality is a convenience which does not affect the generality of the results).

$I$ Identity operator in $\mathfrak{G}_{2}$.

$I_{\mathfrak{W}}$ Identity in $\mathfrak{Q}$ (when it is obvious that we require the restriction of $I$ to $\mathfrak{S}$ we shall drop the subscript).

$P_{\mathfrak{W}}, P_{\mathfrak{G}}$, Projections with range $\mathfrak{L}, \mathfrak{Q}^{\prime}$ respectively.

$V_{\mathfrak{1}} \equiv\left(P_{\mathfrak{W}} H^{\mathfrak{t}}+P_{\mathfrak{G}^{\prime}} S\left(I_{\mathfrak{G}}-H\right) P_{\mathfrak{G}}\right.$ operator whose restriction to $\mathfrak{W}$ as domain is isometric.

$\mathfrak{u}$ Range of $V_{1}$.

$V_{\mathfrak{2}} \equiv\left(-P_{\mathfrak{S}}\left(I_{\mathfrak{G}}-H\right)^{\mathfrak{t}}+P_{\mathfrak{W}^{\prime}} S H \mathfrak{t}\right) S P_{\mathfrak{Q}^{\prime}}$ operator whose restriction to $\mathfrak{S}^{\prime}$ as domain is isometric.

$\mathfrak{u}^{\prime}$ Range of operator $V_{2}$.

$P_{\mathfrak{u}}, P_{\mathfrak{u}}$ Projections with range $\mathfrak{u}, \mathfrak{u}^{\prime}$ respectively.

$P_{1}$ Restriction of $P_{\mathfrak{u}}$ to domain $\mathfrak{q}$.

$V=V_{1}+V_{2}$.

$f, g, \cdots$ Elements of $\mathfrak{Q}_{2}$.

$x, y, \cdots$ Elements of $\mathfrak{k}$.

$x^{\prime}, y^{\prime}, \cdots$ Elements of $\mathfrak{S}^{\prime}$.

$f=x+x^{\prime}$ for all $f \in \mathfrak{S}_{\mathfrak{g}}$ with $x=P_{\mathfrak{S}} f, x^{\prime}=P_{\mathfrak{S}^{\prime}} f . x+x^{\prime}$ will be called the resolved expression for $t$.

It is evident by inspection that $V_{1}$ and $V_{2}$ are isometric if restricted to $\mathfrak{S}$ and $\mathfrak{S}^{\prime}$ respectively and hence $\mathfrak{U}, \mathfrak{u}$ ' are closed. We prove that their ranges are orthogonal. Let $f$ and $g$ be any elements of $\mathfrak{E}_{2}$ and let their resolved expression be $x+x^{\prime}$ and $y+y^{\prime}$. Then

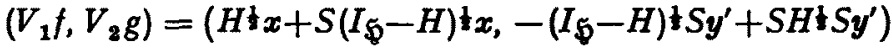

$$
\begin{aligned}
& =-\left(\left(I_{\mathfrak{g}}-H\right) ! H \mathfrak{t} x, S y^{\prime}\right)+\left(H^{\frac{1}{2}}\left(I_{\mathfrak{q}}-H\right) \mathfrak{t} x, S y^{\prime}\right) \\
& =0
\end{aligned}
$$

since $\left(I_{\mathfrak{F}}-H\right)^{\mathfrak{t}}$ and $H^{\mathfrak{t}}$ commute. We have also used the fact that $\left(I_{\mathfrak{G}}-H\right) \mathfrak{t}$ is self-adjoint which is true since the bound of $H$ is less than 1 .

To prove $V=V_{1}+V_{2}$ unitary similar standard arguments are used (Cf. [4] p. 74-5).

It can similarly be proved that $P_{\mathfrak{u}}$ can be expressed explicitly by

$$
P_{\mathfrak{U}}=V_{\mathfrak{1}} H \mathfrak{k} P_{\mathfrak{g}}+V_{\mathfrak{1}}\left(I_{\mathfrak{g}}-H\right) \mathfrak{t} S P_{\mathfrak{G}^{\prime}}
$$

(Cf. [4] p. 75-6).

It follows by inspection that

$$
H=P_{\mathfrak{q}} P_{\mathfrak{u}} P_{\mathfrak{b}}
$$

or what is the same thing, for any element $x$ of $\mathfrak{D}$ 


$$
H x=P_{\mathfrak{Q}} P_{\mathfrak{u}} x .
$$

We shall need some lemmas.

Lemma 14.1. Let $\mathfrak{D}_{A}$ denote the domain of $A$ which is dense in $\mathfrak{W}$. Then $P_{\mathfrak{U}}\left(\mathfrak{D}_{A}\right)$ is dense in $\mathfrak{U}$. Both $\mathfrak{U} \cap \mathfrak{G}$ and $\mathfrak{U} \cap \mathfrak{g}^{\prime}$ are reduced to [0].

Suppose $f \in \mathfrak{U}$ and $f$ orthogonal to $P_{\mathfrak{u}}\left(D_{A}\right)$. Then for all $x \in \mathbb{D}_{A}$

$$
\left(f, P_{\mathfrak{u}} x\right)=0 .
$$

But $f=V_{1} y$ for some $y$ of $\mathfrak{g}$ and the contraction of $P_{\mathfrak{u}}$ on $\mathfrak{G}$ is $P_{1}=V_{1} H^{\mathfrak{t}}$ so that

and hence

$$
\left(V_{1} y, V_{1} H^{t} x\right)=0
$$

$$
\therefore\left(y, H^{\frac{1}{2}} x\right)=\left(H^{\frac{1}{t}} y, x\right)=0
$$

for all $x \in \mathscr{D}_{A}$ and hence $H^{t} y=y=0$. The last remark follows from the fact that both $H^{\mathbf{t}}$ and $\left(I_{\mathfrak{W}}-H\right)^{\mathbf{t}}$ are strictly positive definite.

We have also the following remark which we write as

LEMMA 14.2. The manifold $\mathfrak{u}$ is the graph of the operator $T=(I-H)^{\frac{1}{t}} H^{-\frac{1}{2}}$ if $\mathfrak{G}$ is regarded as the domain space and $\mathfrak{g}^{\prime}$ as the range space.

This is obvious if we recollect that $\mathfrak{u}$ is the range of $V_{1}$. Hence if $f \in \mathfrak{U}$ then

$$
f=V_{1} z
$$

for some $z \in \mathfrak{W}$; it follows that

$$
\begin{aligned}
x=P_{\mathfrak{g}} f & =H^{\frac{1}{2}} z \\
T x=P_{\mathfrak{q}^{\prime}} f & =(I-H)^{\frac{1}{2}} z \\
& =(I-H)^{\frac{1}{2}} H^{\mathfrak{t}} x .
\end{aligned}
$$

It follows from this that $H^{-\frac{1}{d}}$ is unbounded if and only if $\mathfrak{u}$ is asymptotic to $\mathfrak{S}^{\prime}$, i.e. if for every $\varepsilon>0$ there exist vectors $x \in \mathfrak{U}, y \in \mathfrak{S}^{\prime}$ such that $\|x\|=\|y\|=1,\|x-y\| \leqq \varepsilon$ but $\mathfrak{u} \cap \mathfrak{E}^{\prime}=[0]$.

We now proceed to define an operator $K$ in $\mathfrak{U}$ which is to be related to $A$ as follows.

Let $f=P_{\mathfrak{u} x}$ for any $x \in \mathfrak{D}_{A}, A$ symmetrisable by $H$, then

$$
K f=P_{\mathfrak{u}} A x
$$

We now proceed to discuss the properties of $K$. We begin with

Lemma 14.3. If $K$ is defined by (14.4) then it is a symmetric operator in $\mathfrak{A}$.

The domain of $K$ is clearly $P_{\mathfrak{u}}\left(\mathscr{D}_{A}\right)$ which is dense in $\mathfrak{u}$ by lemma 14.1. Let $f$ and $g$ be any two elements of $P_{\mathfrak{u}}\left(D_{A}\right), f=P_{\mathfrak{u}} x$ and $g=P_{\mathfrak{u} y}$ say. Th $\mathfrak{n}$ 


$$
\begin{aligned}
(K f, g) & =\left(P_{\mathfrak{u}} A x, P_{\mathfrak{u} y}\right) \\
& =\left(P_{\mathfrak{u}} A x, y\right) \\
& =\left(P_{\mathfrak{u}} A x, P_{\mathfrak{g}} y\right) \\
& =\left(P_{\mathfrak{g}} P_{\mathfrak{u}} A x, y\right) \\
& =(H A x, y) \\
& =(x, H A y) \\
& =\left(x, P_{\mathfrak{g}} P_{\mathfrak{u}} A y\right) \\
& =(f, K g)
\end{aligned}
$$

Before investigating whether $K$ is maximal and under what conditions $K$ is self-adjoint we show how $K$ is related to $A^{*}$.

THEOREM 14.1. If $K$ is defined by (14.4) then the relations

$$
\begin{aligned}
u & =P_{\mathfrak{Q}} f, \quad f \in \mathfrak{D}_{K} \\
A^{+} u=v & =P_{\mathfrak{G}} K f
\end{aligned}
$$

defined the operator $A^{+}$whose domain is $H\left(\mathfrak{D}_{A}\right)$ which is dense. Also $A^{+}=A^{*}$.

We can put $f=P_{\mathfrak{u} x}$ where $x \in \mathscr{D}_{A}$. Then

$$
\begin{aligned}
u & =P_{\mathfrak{Q}} P_{\mathfrak{u}} x \\
& =H x \\
A+u & =P_{\mathfrak{q}} P_{\mathfrak{u}} A x \\
& =H A x
\end{aligned}
$$

and

$$
A+H x=H A x
$$

in accordance with Definition 8.1. for all $x$ belonging to $\mathfrak{D}_{A}$. The last two statements in the theorem follow from lemma 8.3 and Remark 8.1.

Remark 14.1. (14.4), the definition of $K$ implies

$$
K=V H^{\frac{1}{2}} A H^{-\frac{1}{2}} V^{*}=V H^{-\frac{1}{2}} H A H^{-1} V^{*} \text {. }
$$

It follows that $K$ is closed if and only if $H^{-\frac{1}{3}} H A H^{-1}$ is closed. By virtue of the fact that $H A$ is closed and $H^{-\frac{1}{2}}$ is bounded below it follows that $H^{-1} H A$ is closed (Cf. Dixmier [1] Proposition 3.3). It follows by Dixmier's theorem 3.5 that

LEMMA 14.4. If $K$ is defined by (14.4) it is closed if and only if $H^{\mathrm{t}} x_{n} \rightarrow 0$ and $H \downarrow A x_{n}=H^{-\frac{1}{2} H A x_{n} \rightarrow 0}$ implies $x_{n} \rightarrow 0$.

We now come to discuss possible extensions of $K$.

Lemma 14.5. $K$ cannot have any elements of extension in $P_{\mathfrak{u}}(\mathfrak{S})$.

For if $g \in P_{\mathfrak{u}}(\mathfrak{b})$ then $g=P_{\mathfrak{u} y}$ for some $y$ and for all $x \in \mathfrak{D}_{A}$ 


$$
\left(K P_{\mathfrak{u}} x, P_{\mathfrak{u}} y\right)=\left(P_{\mathfrak{u}} x, K^{*} P_{\mathfrak{u}} y\right)
$$

which by the reasoning of the proof of Lemma 14.3 implies

$$
(H A x, y)=\left(x, P_{\mathfrak{g}} K^{*} P_{\mathfrak{u} y}\right)
$$

for all $x$ belonging to $\mathscr{D}_{A}$ so that since $H A$ is self-adjoint $y \in \mathscr{D}_{A}$ and $P_{\mathfrak{g}} K^{*} P_{\mathfrak{u} y} y=H A y=P_{\mathfrak{g}} K P_{\mathfrak{u}} y$.

We now discuss the nature of possible extensions of $K$ and the relationship of such extensions to $A$ and $A^{*}$. The discussion is exploratory but contains a proof of the statements in theorem 14.2.

By Lemma $14.4 K$ may not be closed. If $P_{\mathfrak{u}}(\mathfrak{g}) \neq \mathfrak{U}$, we can have

$$
\begin{aligned}
P_{\mathfrak{u} x_{n}} & =f_{n} \rightarrow f \\
K f_{n} & \rightarrow g, \quad f \notin \mathfrak{D}_{K} .
\end{aligned}
$$

If $f$ does not belong to $P_{\mathfrak{u}}\left(D_{A}\right), K$ is not defined there. But we have seen that (14.6) is actually impossible for $f$ in $P_{\mathfrak{u}}(\mathfrak{W})$. It follows that we can extend $K$ to a closed symmetric operator $\tilde{K}=K^{* *}$ such that the restriction of $\bar{K}$ on $P_{\mathfrak{u}}(\mathfrak{Q})$ is $K$ as defined by (14.4). It is evident that $P_{\mathfrak{W}} f \in \mathscr{D}_{A *}$. For

$$
\begin{gathered}
f_{n} \rightarrow f \text { implies } P_{\mathfrak{g}} f_{n} \rightarrow P_{\mathfrak{g}} f \\
K f_{n} \rightarrow g \text { implies } P_{\mathfrak{W}} K f_{n}=A^{+} P_{\mathfrak{g}} f_{n} \rightarrow P_{\mathfrak{S}} g
\end{gathered}
$$

which shows that $P_{\mathfrak{S}} f \in \mathscr{D}_{A^{*}}$ and $A^{*} P_{\mathfrak{\$}} f=P_{\$ g}$.

If $\bar{K}$ is not maximal then $\bar{K}$ is clearly not self-adjoint and $\tilde{K} \neq \tilde{K}^{*}$. It is of interest to discover whether $K^{*}\left(=K^{*}\right)$ is related to $A^{*}$.

Let $f$ be any element of $\mathscr{D}_{K}$ and $g$ be an element of $\mathfrak{D}_{K^{*}}$ such that

Then

$$
K^{*} g=h \text {. }
$$

$$
\begin{aligned}
f & =P_{\mathfrak{u} x}=H x+S(I-H)^{\frac{1}{2}} H \frac{\mathfrak{t}}{2} x, \\
K f & =P_{\mathfrak{u}} A x=H A x+S(I-H)^{\frac{1}{2}} H^{\frac{1}{2}} A x .
\end{aligned}
$$

Since $g, h \in \mathfrak{U}$ there exist elements $y, z$ of $\mathfrak{g}$ such that $y=H \mathfrak{\sharp} u, z=H \mathfrak{z} v$ and $g=y+S(I-H)^{\frac{1}{2}} H^{-\frac{1}{2}} y, \quad h=z+S(I-H)^{\frac{1}{2}} H^{-\frac{1}{2}} z$. Now

$$
\begin{aligned}
(K t, g) & =\left(H A x+S(I-H)^{\frac{1}{2}} H^{\frac{1}{2}} A x, y+S(I-H)^{\frac{1}{2}} H^{-\frac{1}{2}} y\right) \\
& =(H A x, y)+((I-H) A x, y) \\
& =(A x, y) \\
\left(f, K^{*} g\right) & =\left(H x+S(I-H)^{\frac{1}{2}} H^{\frac{1}{2}} x, z+S(I-H)^{\frac{1}{2}} H^{-\frac{1}{2}} z\right) \\
& =(H x, z)+((I-H) x, z) \\
& =(x, z) .
\end{aligned}
$$

Hence for all $x \in \mathscr{D}_{A}$

$$
(A x, y)=: \cdots)
$$


so that $z=A^{*} y$. But $y=P_{\mathfrak{S}} g, z=P_{\mathfrak{\Phi}} K^{*} g$ from which we conclude that every element $g$ of $\mathfrak{D}_{R^{*}}$ is such that $P_{\mathfrak{5} g}$ belongs to $\mathfrak{D}_{A^{*}}$ and $P_{\mathfrak{g}} K^{*} g=$ $A^{*} P_{\mathfrak{g}} g$.

Conversely let $y$ be any element of $\mathfrak{D}_{\mathcal{A}^{*}}$ such that $y=P_{\mathfrak{g}}(g), A^{*} y=$ $P_{\mathfrak{u}} h$ where $g, h \in \mathfrak{U}$. Then for all $x \in D_{A}$

$$
(A x, y)=\left(x, A^{*} y\right) \text {. }
$$

Since $A^{*} y=P_{\mathfrak{g}} h, A^{*} y \in \Re_{H^{\ddagger}}$ and

$$
h=A^{*} y+S(I-H)^{\frac{1}{2}} H^{-\frac{1}{2}} A^{*} y .
$$

Then for every $f \in \mathscr{D}_{K}$ and hence $f=P_{\mathfrak{u}} x$, say,

$$
\begin{aligned}
(K f, g) & =\left(H A x+S(I-H)^{\frac{1}{1}} H^{\frac{1}{2}} A x, y+S(I-H)^{\frac{1}{2}} H^{-\frac{1}{2}} y\right) \\
& =(H A x, y)+((I-H) A x, y) \\
& =(A x, y), \\
(f, h) & =\left(H x+S(I-H)^{\frac{1}{1}} H^{\frac{1}{2}} x, A^{*} y+S(I-H)^{\frac{1}{3}} H^{-\frac{1}{2}} A^{*} y\right) \\
& =\left(H x, A^{*} y\right)+\left((I-H) x, A^{*} y\right) \\
& =\left(x, A^{*} y\right) \\
& =(A x, y) .
\end{aligned}
$$

Hence $K^{*} g=h$ and we have

THEOREM 14.2. If $A$ and $K$ are related as in (14.4) then to every $g$ belonging to $\mathfrak{D}_{K^{*}}$ there corresponds a y belonging to $\mathfrak{D}_{A^{*}}$. such that $P_{5} g=y$, $P_{\mathfrak{S}} K^{*} g=A^{*} y$ and to every $y$ in $D_{A^{*}}$ which is such that $y$ and $A^{*} y$ belong to $P_{\mathfrak{Q}}(\mathfrak{U})$ there corresponds a $g$ such that $P_{\mathfrak{Q}} g=y$ and $g$ belongs to $\mathfrak{D}_{K^{*}}$ and $P_{\$} K^{*} g=A^{*} y$.

Remark 14.2. It follows from this that $K^{*}=V H^{-1} A^{*} H^{1} V^{*}$.

We are now in a position to prove

THEOREM 14.3. If $A$ is symmetrisable then $K$ as defined by (14.4) is essentially self-adjoint, i.e. $\tilde{K}=K^{* *}=K^{*}$ in $\mathfrak{u}$.

If $K$ has a deficiency index other than $(0,0)$ there exists an $f$ in $\mathfrak{u}$ such that $K^{*} f=\lambda f$ for some $\lambda$ with $\mathscr{I}(\lambda) \neq 0$. Let $x=P_{\mathfrak{S}} f$ then by Theorem 14.2.

$$
A^{*} x=\lambda x \text { for some } \lambda \text { with } \mathscr{F}(\lambda) \neq 0
$$

which is impossible by theorem 10.8 . We conclude that $K^{*}=K^{* *}$.

We shall show presently that $K \neq \bar{K}$ in general.

Remurk 14.3. The self-adjointness of $H A$ was only appealed to in the last theorem. Lemma 14.5 depends on the fact that $H A$ is maximal symmetric, the other results are true for any $A$ such that $H A$ is symmetric.

It appears worthwhile to investigate, briefly, the effect of relaxing the 
symmetrisability condition and allowing $H A$ to be merely symmetric. We can for instance prove

THEOREM 14.4. If $A$ such that $H A$ is maximal symmetric then $K$ as defined by (14.4) is such that $\tilde{K}=K^{* *}$ is closed maximal symmetric in $\mathfrak{u}$.

To prove this a slight modification of theorem 10.8 is required. The argument used in the proof of theorem 10.8 stands except for the last six lines. Here we use the fact that if $H A$ is maximal $\because\left\{\left((H A)^{*} x_{m}, x_{m}\right)\right\}$ is either non-negative or non-positive (cf. Stone [7] Theorem 9.6) and this together with $\left|\left(x_{n},(H A)^{*} x_{n}\right)-\lambda\left(H x_{n}, x_{n}\right)\right| \leqq \varepsilon$ for arbitrary $\varepsilon$ and sufficiently large $n$ leads to the conclusion that $A^{*}$ can have eigenvalues only in the upper or the lower half of the complex plane but not in both. By repeating the argument in the proof of Theorem 14.3 it is shown that $K^{*} f=\lambda f$ is only possible for $f \neq 0$ if $\mathscr{I}(\lambda) \geqq 0$ (or $\mathscr{I}(\lambda) \leqq 0$ ). This proves that $\tilde{R}$ is maximal.

Still retaining the generalised definition of symmetrisability we add some further remarks about the relationship between $A$ and $K$. The definition (14.4) is seen to imply on substituting for $P_{\mathfrak{U}}: A=H^{-\frac{1}{2}} V^{*} K V H H^{\mathbf{t}}$. (Under fairly general conditions, as we have seen, we can take $\tilde{K}$ in place of $K$ ). By inspection $V^{*} K V=F$, say, is a symmetric operator in $\mathfrak{\$}$. Clearly, since $V$ is unitary, $F$ is self-adjoint or essentially self-adjoint if and only if $K$ is likewise. In any case

$$
A=H^{-\frac{1}{2}} F H^{\frac{1}{2}}
$$

for some symmetric $F$. If $H A$ is closed, then

$$
H A=H^{\frac{1}{2}} F H^{\frac{1}{2}} .
$$

It appears natural to wonder whether choosing $F$ in such a way that (14.8) is self-adjoint (or maximal symmetric etc.) necessarily leads to symmetrisable $A$ (or $A$ such that $H A$ is maximal symmetric etc.)? The answer is in the negative. For let $F$ be the "elementary symmetric transformation" defined by $i(I+U)(I-U)^{-1}$, where $U$ is the isometric transformation which takes $e_{i}$ into $e_{i+1}$ for some complete orthonormal system $\left(e_{i}\right)$ in $\mathfrak{g}$. Let $H^{\mathfrak{t}}=$ $\frac{1}{4}(I-U)\left(I-U^{*}\right)$. Then

$$
\begin{aligned}
H^{\frac{1}{2}} F H^{\frac{1}{2}} & =\frac{i}{1^{6}}(I-U)\left(I-U^{*}\right)(I+U)(I-U)^{-1}(I-U)\left(I-U^{*}\right) \\
& =\frac{i}{\Gamma^{6}}(I-U)\left(U-U^{*}\right)\left(I-U^{*}\right)
\end{aligned}
$$

which is bounded symmetric and therefore self-adjoint. But since $F$ is only symmetric in $\mathfrak{Q}, K$ is only symmetric in $\mathfrak{U}$ so that by theorem $\mathbf{1 4 . 3}$ $A$ cannot be symmetrisable. It is evident in any case that $A=H^{-1} F H^{\mathbf{t}}$ is unbounded so that $H A$ cannot be equal to $H^{\frac{1}{2}} F H^{\frac{1}{2}}$.

There is a representation of $A^{*}$ equivalent to (14.7). To establish it we use 
Lemma 14.6. If $A$ is closed $B$ bounded and closed and $D_{A B}$ and $D_{A}$ dense then $(A B)^{*}=\overparen{B^{*} A^{*}}$.

By Dixmier [1] Proposition 3.3, $A B$ is closed. Let $(A B)^{*}=C$. For all $x \in \mathscr{D}_{A B}, y \in \mathscr{D}_{C}:\left((A B)^{*} y, x\right)=(y, A B x)$. For all $z$ in the domain of $A^{*}$ and $u$ in the domain of $\left(B^{*} A^{*}\right)^{*}$

$$
\left(B^{*} A^{*} z, u\right)=\left(A^{*} z, B u\right)=\left(z,\left(B^{*} A^{*}\right)^{*} u\right) .
$$

Hence $B u$ is in the domain of $A^{* *}=A$ and $\left(B^{*} A^{*}\right)^{*}=A B$ since always $\left(B^{*} A^{*}\right)^{*} \supset(A B)^{* *}$. Therefore $\left(B^{*} A^{*}\right)^{* *}=(A B)^{*}$ or $(A B)^{*}=\overparen{B^{*} A^{*}}$.

Corollary. If $G$ is also bounded, then $(G A B)^{*}=(A B)^{*} G^{*}=\left(\widetilde{B^{*} A^{*}}\right) G^{*}$.

Proof. For all $x$ in $\mathfrak{D}_{G A B}=\mathscr{D}_{A B}$ and all $y$ in $\mathscr{D}_{(G A B)^{*}}$

$$
\begin{aligned}
\left(x,(G A B)^{*} y\right) & =(G A B x, y)=\left(A B x, G^{*} y\right) \\
& =\left(x,(A B)^{*} G^{*} y\right)=\left(x,\left(\widetilde{B^{*} A^{*}}\right) G^{*} y\right) .
\end{aligned}
$$

We can now construct $A^{*}$ explicitly.

The analogue of the operator $A^{+}$defined in Section 8 for symmetrisable $A$ is defined by $A^{+} H=(H A)^{*}$. If $H A$ is maximal symmetric then the proof of theorem 8.2 together with Remark 8.1 stands with obvious minor modifications so that $\bar{A}^{+}=A^{*}$. By (14.8) and the corollary to lemma 14.6

so that

$$
(H A)^{*}=\overparen{H^{ \pm} F^{*}} H^{\frac{1}{2}}
$$

and

$$
A^{+}=\left(\widetilde{H^{\frac{1}{1} F^{*}}}\right) H^{-\frac{1}{2}}
$$

since the range of $H^{-\frac{1}{2}}$ is $\Re$.

$$
\begin{aligned}
& A^{*}=\widehat{\left(\widehat{\left.H^{\frac{1}{2} F^{*}}\right) H^{-\frac{1}{2}}}\right.} \\
& =\widehat{H^{\frac{1}{2}} F^{*} H^{-\frac{1}{2}}}
\end{aligned}
$$

\section{Remarks on the spectrum}

The construction of section 14 can be expected to throw some light on the continuous and the residual spectrum of $A$. If the operator $K$ is selfadjoint, i.e. $A$ is symmetrisable, then every $f$ of $\mathfrak{u}$ is in the range of $\tilde{K}-\lambda I$ for all non-real $\lambda$. Let $P_{1}$ be the restriction of $P_{\mathfrak{u}}$ to the domain $\mathfrak{g}$ then $\lambda$ will belong to the resolvent set of $A$ if and only if for all $f$ in the range of $P_{1}$ there exists a $g$ in the range of $P_{1}$ such that

$$
(\tilde{K}-\lambda I) g=f \text {. }
$$

It can be observed immediately that if the domain or range of $\tilde{K}$ is contained 
in the range of $P_{1}$ then a non-real $\lambda$ does not belong to the continuous spectrum of $A$. For if $f=P_{1} x$ and either $g$ or $K g$ belong to $P_{1}(\mathfrak{G})$ then the other one must also, and in consequence $g=P_{1} y$ and the range of $P_{1}^{-1}(\tilde{K}-\lambda I)$ i.e. $(A-\lambda I)$ is $\mathfrak{G}$. The same argument shows that any $\lambda$ in the resolvent set of $\tilde{R}$ is in the resolvent set of $A$.

We have, however, the following

Remark 15.1. The condition $\mathfrak{D}_{R} \subset \mathfrak{R}_{P_{1}}$ implies $P_{1}(\mathfrak{S})=\mathfrak{u}$ which implies $H^{-\frac{1}{2}}$ is bounded. For $\mathfrak{D}_{\tilde{K}} \subset \Re_{P_{1}}$ implies $K=\tilde{K}$ by lemma 14.5 and $\Re_{K} \subset \Re_{P_{1}}$ by definition (14.4); but $\mathfrak{D}_{K} \cup \mathfrak{R}_{K}=\mathfrak{U}$ when $K$ is self-adjoint ${ }^{1}$ so that $\mathfrak{R}_{P_{1}} \supset \mathfrak{U}$; by definition (14.1) $\mathfrak{R}_{P_{1}}=V_{1}\left(\mathfrak{R}_{H^{\mathfrak{t}}}\right)$ so that $\mathfrak{R}_{H^{\ddagger}}=V^{*}(\mathfrak{U})=\mathfrak{S}$. (A more general statement is contained in lemma 15.1).

The above remarks suggest that it may be profitable to study operators $K$ or $\widetilde{K}$ of the form $V H^{\frac{1}{2}} B H^{\frac{1}{2}} V^{*}$ where $B$ is at least symmetric. We shall call those $K$ of type $P$. By Remark 14.1 such operators correspond to operators $A$ for which $B H^{\frac{1}{2}}=A H^{-\frac{1}{2}}$ and hence $A=B H$. (By Corollary to lemma 14.6 such an $A$ is symmetrisable (by $H$ ) if $H B H=\left(H B^{*}\right) H$. When $\tilde{K}$ is of type $P$, we have $\Re_{K} \subset \Re_{P_{1}}$ so that the resolvent set of $\bar{K}$ is the resolvent set of $A$, the continuous spectrum is real and the residual spectrum empty. An example of such operators are operators of the type $A=B H$ for which $B$ is bounded. ( $K \neq R$ in general, in this case.)

$A=B H$ may be symmetrisable for $B$ merely symmetric as is shown by the following example. Let $U$ be the isometric transformation defined towards the end of section 14 (i.e. $U e_{i}=e_{i+1}$ ). Let $B=i(I+U) /(I-U)$, $H=\frac{1}{4}(I-U)\left(I-U^{*}\right)$ then $A=\frac{i}{4}(I+U)\left(I-U^{*}\right), H A=\frac{i}{16}(I-U)\left(U-U^{*}\right)$ $\left(I-U^{*}\right)$, which is self-adjoint. Both $A$ and $H A$ are bounded. It can be easily verified that $K=V H^{\frac{1}{2}} B H^{\frac{1}{2}} V^{*}$ is unbounded and that $i$, but not $-i$, belongs to the continuous spectrum of $A$. Clearly $K$ is not closed and $\vec{K}$ is not of type $P$. This is another example of symmetrisable operators with a nonreal continuous spectrum.

When $\tilde{R}$ is merely symmetric, i.e. $A$ such that $H A$ is symmetric, then for some $g \in \mathfrak{U}$ and some $\lambda$, with $\mathscr{I}(\lambda) \neq 0, K^{*} g=\lambda g$. It follows from theorem 14.2 that $y=P_{\xi g}$ is an eigen-element of $A^{*}$ and $A^{*} g=\lambda g$ and consequently $\bar{\lambda}$ is in the residual spectrum of $A$. This reasoning can be extended.

THEOREM 15.1. If $A^{*}$ has an eigenvalue $\lambda$ with $\mathscr{I}(\lambda)>0(<0)$ and with eigen-element $y$ in $\Re_{H}$ then the whole half-plane $\mathscr{I}(\lambda)<0(>0)$ belongs to the residual spectrum of $A$.

\footnotetext{
1 It is evident that since the graphs of $K$ and $-K^{-1}\left(K^{-1}\right.$ may be multivalued in this context) are orthogonal complements the sum of the projections of these graphs onto the domain space must be the whole domain space. (Cf. Dixmier [1] Remark (R), in particular equation (2) p. 19.)
} 
For any $y=H \mathfrak{t} x$ we have, $g=V_{1} x$ where $g \in \mathfrak{U}$ and $y=R_{\$} g$. Hence $g$ is a characteristic element of $K^{*}$ with characteristic value $\lambda$ where $f(\lambda)>0$ $(<0)$. By the properties of the symmetric operator $K$ it then follows that the whole half-plane $\mathscr{I}(\lambda)>0(<0)$ is in the point spectrum of $K^{*}$ and hence of $A^{*}$.

For symmetric $K$ it may be worth-while to make some comments on the case when $K$ is closed. The range of $(K-\lambda I)$ is contained (in the wide sense) in the range of $P_{1}$. Hence if the range of $H^{t}$ is not closed then for all $\lambda$ there are elements of $\mathfrak{u}$ not in the range of $K_{\lambda}$, i.e. the resolvent set is empty. It follows that $K$ cannot be maximal symmetric for in that case one of the half-planes $\mathscr{f}(\lambda)>0(<0)$ would belong to the resolvent set. This, in conjunction with theorem 15.1 leads to

LEMMA 15.1. If $A$ is an operator such that $H A$ is maximal symmetric then the corresponding $K$ as defined in (14.4) is closed only if $H^{-1}$ is bounded.

Lemma 14.4 is therefore trivial for all $A$ such that $H A$ is maximal symmetric. The remarks made relating the continuous spectrum of $A$ to the range of $K$ apply, with obvious modifications, to the case when $H A$ is merely symmetric.

The results obtained about the spectrum of symmetrisable operators are summarised in

THEOREM 15.2. Let $A$ be a symmetrisable operator in $\mathfrak{\mathfrak { E }}, H$ its symmetrising operator, $K$ the operator in $\mathfrak{U}$ defined by (14.4) and $P_{1}$ the restriction of $P_{\mathfrak{U}}$ to domain $\mathfrak{G}$. Then

(i) the point spectrum of $A$ and $A^{*}$ (if it exists) is real, the point spectrum of $A^{*}$ contains the point spectrum of $A$;

(ii) $K$ is self-adjoint; $K=\mathbb{K}$ if and only if $H^{-1}$ is bounded;

(iii) if $(\widetilde{K}-\lambda I) f \in \Re_{P_{1}}$ implies $f \in \Re_{P_{1}}$ then the whole spectrum of $A$ is restricted to the real axis and the residual spectrum is empty;

(iv) there exist symmetrisable operators for which the continuous spectrum is not restricted to the real axis;

(v) by (i) the residual spectrum of $A$ cannot include $\lambda$ if $\mathscr{I}(\lambda) \neq 0$;

(vi) class (iii) includes all $A$ for which $\Re_{R} \subset \mathscr{I}_{P_{1}}$ in particular class (ii) and all operators $A=B H$ where $B$ is bounded, self-adjoint.

A similar theorem could be stated for $A$ such that $H A$ is merely symmetric.

\section{Extension of the space}

It was mentioned in section 9 that a possible way of dealing with a symmetrisable operator $A$ in $\mathfrak{F}$ is to introduce in $\mathfrak{\mathscr { D }}$ a new inner product and 
hence a new metric which would make $A$ symmetric. This procedure will now be described in detail. We continue to assume the symmetrising operator $H$ to be positive, self-adjoint and have bound less than 1 .

Let $x_{n} \in \mathfrak{R}_{H^{+}}$and $f_{n}=H^{-1} x_{n}$. Let $\mathfrak{Q}^{+}$be the linear space consisting of all elements such as $f_{n}$ (i.e. $\mathfrak{E}$ ) and possibly certain others. In $\mathfrak{Q}^{+}$an inner product is defined by

$$
(f, g)_{+}=\left(H^{\frac{1}{2}} f, H^{\frac{1}{2}} g\right)
$$

to begin with for all $f, g$ in $\mathfrak{S}$; this also supplies the metric for $\mathfrak{S}^{+}$. Then $\mathfrak{S}^{+}$ satisfies all the Hilbert space axioms except possily completeness. The extension of $\mathfrak{g}$ follows standard lines so we shall only sketch the procedure. If $H \nmid$ has no positive lower bound then $\Re_{H t}$ is not closed although it is everywhere dense. Let $x$ be an element of $\mathfrak{g}$ not beloning to $\Re_{u^{t}}$. Let $\left(x_{n}\right)$ be a sequence of elements of $\Re_{H t}$ converging to $x$. Let $f_{n}=H^{-\frac{1}{2}} x_{n}$. Then

$$
\begin{aligned}
\left\|f_{n}-f_{m}\right\|_{+}^{2} & =\left(H \mathbf{z}\left(f_{n}-f_{m}\right), H \mathbf{t}\left(f_{n}-f_{m}\right)\right) \\
& =\left\|x_{n}-x_{m}\right\|^{2} \rightarrow 0 \text { as } n, m \rightarrow \infty .
\end{aligned}
$$

Therefore the sequence $\left(f_{n}\right)$ converges in $\mathfrak{S}^{+}$but it cannot converge to an element of $\mathfrak{W}$ since $x \notin \mathfrak{R}_{H}$ and $H \mathfrak{t}$ is closed in $\mathfrak{W}$. Hence we must add an ideal element $f^{*}$ to $\mathfrak{W}$ so that $\mathfrak{S}^{+} \supset \mathfrak{W} \cup\left\{f^{*}\right\}$, if $\mathfrak{S}^{+}$is to be complete. Furthermore $H^{t}$ is extended to $f^{*}$ by putting $H \ddagger f^{*}=x$ and then (16.1) is extended to $\left\{f^{*}\right\}$. This process of extension is carried out for all sequences $\left(f_{n}\right)$ which converge in the $\mathfrak{G}^{+}$metric. If this is done $\mathfrak{G}^{+}$is complete. For suppose there exists a sequence containing ideal elements $f_{n}^{*}$ such that $\left\|f_{n}^{*}-f_{m}^{*}\right\|_{+} \rightarrow 0$ as $n, m \rightarrow \infty$. Suppose the limit of the sequence $f_{n}^{*}$ is $g^{*}$ which does not belong to $\mathfrak{S}^{+}$. Now for every $f_{n}^{*}$ there exists a sequence $\left(f_{n, p}\right)$ of elements of $\mathfrak{E}$ such that $\lim _{p \rightarrow \infty}\left\|f_{n, p}-f_{n}^{*}\right\|_{+} \rightarrow 0$ so that

$$
\left\|f_{n, s}-f_{m, \theta}\right\|_{+} \leqq\left\|f_{n, s}-f_{n}^{*}\right\|_{+}+\left\|f_{n}^{*}-f_{m}^{*}\right\|_{+}+\left\|f_{m}^{*}-f_{m, a}\right\|_{+}<\varepsilon
$$

if $m, n, p, q$ are large enough. Hence a simple sequence can be picked out of the double sequence $\left(f_{n, p}\right)$ which converges to an element of $\mathfrak{Q}^{+}, f^{*}$ say. Clearly $f_{n}^{*}$ also converges to $f^{*}$ and $g^{*}=f^{*}$. It follows that $\mathfrak{G}^{+}$is complete and $\mathfrak{G}$ is dense in $\mathfrak{S}^{+}$(in the $\mathfrak{Q}^{+}$topology). The extended $H^{\mathfrak{t}}$ is everywhere defined on $\mathfrak{Q}^{+}$and its range is $\mathfrak{W}$. Also for all $f, g \in \mathfrak{Q}^{+}$

and

$$
\begin{aligned}
& \|H f\|_{+}^{2}=(H f, H f)_{+}=\left(H^{\prime} f, H^{\frac{1}{2}} f\right) \leqq\left\|H^{2} H^{\mathbf{b}} f\right\|\left\|H^{\mathbf{b}} f\right\| \\
& <\| H^{\mathfrak{t} f \|^{2}} \quad \text { (since } H \mathbf{t} t \in \mathfrak{W} \text { ) } \\
& =\|f\|_{+}^{2}
\end{aligned}
$$

$$
(H f, g)_{+}=\left(H^{\frac{1}{1}} H f, H^{\mathbf{t} g}\right)=\left(H^{\mathbf{l}} f, H H^{\frac{1}{2}} g\right)=(f, H g)_{+} ;
$$

further 


$$
\begin{aligned}
\left\|H f^{*}\right\|_{+}=0 & \Rightarrow\left(H H^{\frac{1}{2}} f^{*}, H H^{\frac{1}{2}} f^{*}\right)=0 \Rightarrow H^{\frac{1}{2}} f^{*}=0 \\
& \Rightarrow\left(H^{\frac{1}{2}} f^{*}, H^{\frac{1}{2}} f^{*}\right)=\left\|f^{*}\right\|_{+}=0
\end{aligned}
$$

so that $H$ has bound less than 1 , is self-adjoint and positive definite.

All the operators like $V_{1}, V_{2}, P$ defined at the beginning of section 14 were defined explicitly in terms of $H$. Therefore they can be extended by merely extending $H$ since $H$ still has the same bound.

Let $A$ be any operator symmetrisable by $H$. Its definition in $\mathfrak{g}$ ensures that it is properly defined in $\mathfrak{S}^{+}$for

LEMMA 16.1. If $\mathfrak{D}$ is dense in $\mathfrak{S}$ it is also dense in $\mathfrak{\mathfrak { Q }}^{+}$.

Suppose the lemma false. Then there exists an element $f^{*}$ in $\mathfrak{S}^{+}$such that $\left(x, f^{*}\right)_{+}=0$ for all $x \in \mathfrak{D}$. But this implies, since $H f^{*} \in \mathfrak{H}$,

$$
\left(x, H f^{*}\right)=0 \text { for all } x \in \mathbb{D} \text {, }
$$

which is impossible if $\mathfrak{D}$ is dense in $\mathfrak{Q}$ unless $f^{*}=0$.

Hence $A$ is defined on a domain dense in $\mathfrak{S}^{+}$. Further if $f, g \in \mathfrak{D}_{A}$ then

$$
(A f, g)_{+}=(H A f, g)=(f, H A g)=(f, A g)_{+}
$$

so that $A$ is symmetric in $\mathfrak{Q}^{+}$, in the $\mathfrak{S}^{+}$-topology. We must introduce a distinctive notation for the adjoint in $\mathfrak{Q}^{+}$and we put $T_{+}^{*}$ to denote the adjoint of $T$, viz.

$$
(T f, g)_{+}=\left(f, T_{+}^{*} g\right)_{+}
$$

where this relation is defined. When $f \in \mathfrak{E}$ this leads to

$$
(T f, H g)=\left(f, H T_{+}^{*} g\right)
$$

so that $T^{*} H \supset H T_{+}^{*}$ with equality when $T$ is defined only in $\mathfrak{Q}$. Wher $A$ is symmetrisable in $\mathfrak{W}$ (and only defined in $\mathfrak{G}$ ) $H A_{+}^{*}=A^{*} H$ or $A_{+}^{*}=A$ on $\mathfrak{W}$. If $A$ is not closed or not self-adjoint in $\mathfrak{W}^{+}$then the elements of extension must be ideal elements. This is because $f_{n} \stackrel{+}{\rightarrow} f, A f_{n} \stackrel{t}{\rightarrow} g$, where $f_{n}, f$ belong to $\mathfrak{W}$, would imply not only

$$
\left(A x, f_{n}\right)_{+}=\left(x, A f_{n}\right)_{+} \text {for all } x \in \mathscr{D}_{A} \text {, and for all } n \text {, }
$$

but in particular going to the limit

$$
(H A z, f)=(x, H g)
$$

and $(H A)^{*} f=H A f=H g$, since $H A$ self-adjoint in $\mathfrak{Q}$ and hence $f \in D_{A}$.

However, results such as these can be obtained from the analysis of section 14. For it follows from the fact that $H^{\mathfrak{l}}\left(\mathfrak{Q}^{+}\right)=\mathfrak{W}$ and $P_{\mathfrak{u}}\left(\mathfrak{Q}^{+}\right)=$ $V_{1} H^{1}\left(\mathfrak{\Phi}^{+}\right)=V_{1}(\mathfrak{W})=\mathfrak{u}$ that the operator $P_{\mathfrak{u}}$ with domain restricted to $\mathfrak{\mathfrak { W }}^{+}$defines a one-one correspondence between all of $\mathfrak{\Phi}^{+}$and all of $\mathfrak{U}$. Therefore relations such as (14.4) will define operators in $\mathfrak{l}$ by operators in $\mathfrak{W}^{+}$ 
and conversely. Further by using the relations $A_{+}^{*}=H^{-1} A^{*} H$, and $K=$ $V H^{\frac{1}{2}} A H^{-\frac{1}{2}} V^{*}, K^{*}=V H^{-\frac{1}{2}} A^{*} H^{\frac{1}{2}} V^{*}$ with the extended definitions of $V$ and $H^{t}$ it is seen that $K$ and $K^{*}$ correspond to $A$ and $A_{+}^{*}$. Hence if $K$ is symmetric or self-adjoint in $\mathfrak{U}, A$ is symmetric or self-adjoint in $\mathfrak{G}^{+}$. Further if $K$ has no extensions on $P_{\mathfrak{u}}(\mathfrak{Q}), A$ has no extensions on $\mathfrak{G}$. Also if $K$ is closed in $\mathfrak{U}, A$ is closed in $\mathfrak{G}^{+}$since a sequence $f_{n}$ converges in $\mathfrak{T}^{+}$if and only if $P f_{n}$ converges in $\mathfrak{U}$. Finally by theorem 14.3.

THEOREM 16.1. If $A$ is symmetrisable in $\mathfrak{S}$ then $A$ is essentially selfadjoint in $\mathfrak{E}^{+}$.

The approach to symmetrisable operators by considering them in the extended space $\mathfrak{S}^{+}$(in the $\mathfrak{S}^{+}$-topology) was essentially the method used by K. O. Friedrichs [2] in his analysis of semi-bounded operators. By the above remarks an analysis of operators in $\mathfrak{S g}^{+}$is equivalent to an analysis of corresponding operators in $\mathfrak{u}$. In sections 14 and 15 an attempt was made to relate the properties of such operators to those of operators in $\mathfrak{S}$. It became evident there that the properties that had the most crucial bearing on the basic operator $A$ in $\mathfrak{S}$ were the domain and range of the induced operator $\widetilde{K}$ in $\mathfrak{u}^{2}{ }^{2}$ These properties have their exact equivalents in the corresponding operators in $\mathfrak{S}^{+}$(the extensions of $A$ to $\mathfrak{S}^{+}$). The advantage, if any, in considering $K$ in $\mathfrak{u}$ rather than its equivalent in $\mathfrak{g}^{+}$is the transparent relation of $K$ not only to $A$ but also to $A^{*}$, so that properties of the three operators could be studied simultaneously. The relation between the properties of operators $A$ and their extensions in $\mathfrak{W}^{+}$to the properties of $A$ in $\mathfrak{W}$ is exactly analogous to the relation of the properties of $K$ and its extensions to the properties of the corresponding $A$ in $\mathfrak{b}$.

\section{Acknowledgement}

As mentioned before, the author feels greatly indebted to Dr. F. Smithies for encouragement, suggestions and criticism, and to the Australian C.S.I.R. and Department of Supply for financial assistance during the time when this research was originally carried out.

\section{References}

[1] Dixmier, J., Bull. Soc. Math. France, 77 (1949) 11-101.

[2] Friedrichs, K. O., Math. Annaten 109 (1934) 465-487.

[3] von Neumann, J., Functional Operators Vol. II, Annals of Mathematics Studies 22, Princeton 1950 .

- Properties such as boundedness appear to lose their importance, for boundedness in $\mathfrak{S}^{+}$ does not imply boundedness in $\$$ and conversely, without additional conditions on the relation between $A$ and $H$. 
[4] Silberstein, J. P. O., Dissertation, Cambridge, 1952.

[5] Silberstein, J. P. O., Symmetrisable Operators, This Journal 2 (1961-2), 381-402.

[6] Silberstein, J. P. O., Symmetrisable Operators Part II, This Journal 3 (1963),

[7] Stone, M. H., Linear Transformations in Hilbert Space, Amer. Math. Soc. Colloquium Publications XV, New York 1932.

Department of Mathematics, University of Western Australia. 